\title{
Un nouveau programme en éducation thérapeutique : former des soignants pour favoriser la résilience des patients
}

\author{
A New Program in Therapeutic Education: \\ Training Caregivers to Foster the Resilience of Patients \\ Monique CHAMBOULEYRON ${ }^{1}$, Aline LASSERRE-MOUTET', Stefan VANISTENDAEL' \\ Grégoire LAGGER ${ }^{1}$, Alain GOLAY
}

Résumé Contexte: Dans leur pratique d'entretien, les médecins interrogent les patients pour rechercher des signes pathologiques et des symptômes, et ils recherchent rarement les aspects psychosociaux ainsi que les ressources des patients. L'objectif est d'évaluer si une formation en éducation thérapeutique du patient (ETP) centrée sur les thèmes de la résilience et de l'empowerment, influe sur la pratique des soignants, vers une plus grande part donnée à la recherche des ressources des patients. Population et méthodes : 2 médecins et 3 infirmières ont participé pendant 11 mois à une formation continue centrée sur ces concepts. 6 entretiens d'entrée de patients diabétiques hospitalisés pour une semaine d'éducation thérapeutique, ont été enregistrés avant la formation et 6 autres après. Les interviews ont été analysées par une pédagogue non impliquée dans cette formation. Résultats : Après formation, les soignants recherchent plus les ressources des patients $(2,0 \pm 0,3$ après, $0,1 \pm 0,1$ avant, $p<0.001$ ). Après une réponse du patient, il y a moins d'informations ou d'investigations biomédicales complémentaires (7,3 $\pm 1,4$ après, $18,9 \pm 2,5$ avant, $p<0,001)$, plus de reformulations $(4,3 \pm 0,9$ après, $1,5 \pm 0,4$ avant, $p<0,01)$, plus de mises en lumière des ressources du patient $(1,5 \pm 0,3$ après, $0,4 \pm 0,1$ avant, $p>0,01)$. La durée des interviews n'est pas rallongée malgré plus d'investigations psychosociales et sur les ressources des patients ( $40,8 \pm 3$ après, $38,3 \mathrm{mn} \pm 4$ avant). Conclusions : Cette formation permet aux soignants de mieux repérer les ressources des patients. Plus encore, porter attention à ce qui va bien les amène à passer d'une logique d'interrogatoire à une logique d'incitation des patients à prendre part à la compréhension et la construction de leur propre prise en charge dès la première rencontre.

Mots clés Education thérapeutique du patient ; résilience ; empowerment ; formation soignants.

Abstract Background: In the history part of their clinical encounters, healthcare providers collect data on signs and symptoms, but rarely on illness psychosocial aspects and on patient resources. Objectives: The aim of this study is to evaluate whether a training in therapeutic education centred on patient resilience and empowerment has an influence on health care professional's (HCP) approach, providing a larger part to the investigation of patient resources. Methods: 2 physicians and 3 nurses participated in a training on resilience and empowerment that was carried out over 11 months. 6 interviews - realized before and after training - that participants conducted with diabetic patients hospitalised for a patient education week were recorded. These interviews were analysed by an adult education specialist who was not implicated in the training. Results: HCP ask patients significantly more questions on their resources $(2,0 \pm 0,3$ after, $0,1 \pm 0,1$ before, $p<0,001)$. In the way HCP react to patients' words, there is less instantaneous refocusing on bio-medical subject, giving or looking for further information $(7,3 \pm 1,4$ after, $18,9 \pm 2,5$ before, $p<0,001)$. There are more reformulations $(4,3 \pm 0,9$ after, 1,5 $\pm 0,4$ before, $p<0,01)$ and more emphasizing on patients speech located resources (1,5 $\pm 0,3$ after, $0,4 \pm 0,1$ before, $p<0,01)$. Interviews length was not modified despite an increase of investigations regarding psychosocial aspects and patients' resources (40,8 \pm 3 min. after, 38, $3 \pm 4$ min. before). Conclusions: this study shows that the training allows HCP to better identify patients' resources. Even more, taking care of what goes well make them pass from a logic of data collection to a logic of patient incitation to become active partner of care.

Key Words Therapeutic education; resilience; empowerment; training; health care professional

Pédagogie Médicale 2007;8:199-206

REVUE INTERNATIONALE FRANCOPHONE D'ÉDUCATION MÉDICALE 


\section{Recherche et Perspectives}

\section{Introduction}

L'éducation thérapeutique des patients est une approche humaniste centrée sur le patient ${ }^{1}$. Elle est centrée notamment sur les besoins, les connaissances et les compétences du patient, mais également sur ses ressources². L'éducation thérapeutique du patient est appliquée pour des malades chroniques ; elle élargit l'approche biomédicale habituellement enseignée dans nos facultés par une approche systémique et psychosociale. Elle comprend particulièrement un diagnostic psychopédagogique associé à un diagnostic biomédical conventionnel. Les patients souffrant de maladies chroniques ont très souvent une rupture des sentiments de sécurité et d'identité (powerlessness). Cette perte d'identité est due à un écart de son image entre avant et après la maladie. Elle est aussi induite par un changement des perceptions corporelles et du rôle social. La perte du sentiment de sécurité peut être à la fois corporelle, émotionnelle, sociale, voire financière. Le but de cette étude est d'évaluer l'impact d'une formation de soignants aux concepts et principes de l'éducation thérapeutique du patient, focalisée particulièrement sur la résilience et l'empowerment. Cette formation devrait les amener à aider plus systématiquement les patients à prendre conscience de leurs ressources.

En effet, pour parvenir à un contrôle optimal de leur pathologie, les malades chroniques doivent développer des compétences et des stratégies, à un moment où ils sont physiquement et psychologiquement fragilisés par cette maladie. Cette conjoncture les amène souvent à un découragement et à l'adoption d'une attitude passive dans la prise en charge de leur maladie chronique.

Face à cette problématique, le concept de résilience peut nous apporter des éléments de réflexion intéressants. La résilience peut se définir comme la double capacité à surmonter une épreuve, puis à prendre un nouveau départ dans l'existence. Il s'agit donc de l'association de ces deux tenants : résistance aux traumatismes et dynamique existentielle $e^{4}$. Ce concept a été proposé à partir d'observations d'individus ou groupes d'individus qui, ayant vécu des situations ou évènements difficiles, peuvent rebondir et mieux vivre avec une maladie chronique ou un handicap. Ils reprennent le cours de leur vie de façon favorable, alors même que les autres s'enfoncent vers une issue négative ${ }^{5,6}$. Ces personnes ont su mobiliser leurs ressources individuelles, familiales ou sociales. Vanistendael propose une modélisation de ces ressources sous la forme de la " casita » Le modèle de la résilience, développé dans le domaine psychosocial et de l'enfance, a ensuite été appliqué au monde médical pédiatrique (enfance et adolescence) ${ }^{8,9}$. Il fait aujourd'hui son apparition dans le domaine de la pathologie adulte et des maladies chroniques ${ }^{10}$. Il est vrai qu'un patient n'a pas forcément un traumatisme physique mais il n'est pas rare que la maladie chronique entraîne un traumatisme psychologique, avec une proportion importante de patients souffrant de dépression, anxiété et troubles du comportement. Le concept de résilience est certainement approprié dans le contexte de la maladie chronique ${ }^{11}$.

Ce concept de résilience est séduisant à plusieurs titres ${ }^{12,13}$. Il montre que l'évènement stressant l'est aussi en fonction de l'interprétation qu'en fait la personne et du pouvoir traumatisant qu'elle lui attribue. Il met l'accent sur le fait que l'individu n'est pas une entité autosuffisante et isolée mais qu'il vit dans un milieu familial, professionnel, social, riche en interactions et qu'il est donc primordial de tenir compte du patient centré dans son milieu psychosocial. Il rappelle enfin que la personne puise son énergie et ses capacités dans ses ressources propres et ses modèles de croyance. Une personne ressource, un projet ou un trait de caractère peuvent aider une personne à surmonter les épreuves de la vie.

La notion de ressources est alors centrale dans la capacité de l'individu à " métaboliser » l'événement stressant, et à poursuivre sa vie. Le patient gère donc sa maladie chronique au quotidien grâce à ses propres ressources. Or, il est impossible de s'appuyer sur des ressources dont on n'a pas ou plus conscience. Le patient ne voit souvent plus que ses handicaps et limites.

Le soignant, accompagnateur privilégié de la personne malade, devrait pouvoir l'aider à chercher ses ressources. Il peut jouer un rôle crucial de tuteur de résilience et permettre au patient de s'appuyer sur ses propres ressources. L’anamnèse médicale habituelle est essentiellement basée sur la recherche de critères biomédicaux prédéfinis, permettant de s'orienter efficacement dans une démarche de type diagnostic différentiel et d'approche algorithmique. Par ailleurs elle nous conduit à rechercher les éléments négatifs, pathologiques plutôt que les éléments positifis ${ }^{12-14}$, comme les ressources, trop souvent ignorées. L'anamnèse médicale à but diagnostique devrait se coupler avec une anamnèse psychosociale, permettant également de poser un diagnostic psychopédagogique. Avec ce type d'anamnèse, l'accent peut

\footnotetext{
1- Service d'éducation thérapeutique pour les maladies chroniques (SETMC) - Hôpitaux universitaires de Genève.

2- Bureau international catholique de l'enfance (BICE) - Genève.

Correspondance : Monique Chambouleyron - Service d'éducation thérapeutique pour les maladies chroniques (SETMC) -

Hôpitaux universitaires de Genève - 24 rue Micheli-du-Crest - 1211 Genève 14, Suisse. Téléphone : 0041223729717. Mailto: monique.chambouleyron@hcuge.ch
} 
être mis sur les points forts du patient et mettre en évidence ses propres ressources. Interroger les patients, pour faire émerger leurs ressources et leur en faire prendre conscience, nécessite donc au préalable que les soignants aient été formés à cette approche et aient modifié leur propre culture. En plus, une anamnèse plus globale, biopsychosociale, avec une démarche recherchant en plus les ressources du patient, permet un recentrage thérapeutique plus efficace ${ }^{14}$. L'objectif est d'évaluer si une formation en éducation thérapeutique du patient (ETP) centrée sur les thèmes de la résilience et de l'empowerment influe la pratique des soignants, vers une plus grande part donnée à la recherche des ressources des patients.

\section{Population et méthodes Population}

Cinq soignants ( 2 médecins et 3 infirmières) ont été évalués avant la formation puis 11 mois plus tard. Les deux médecins étaient sans formation préalable à l'éducation thérapeutique alors que les infirmières avaient une expérience de 2,8 $\pm 0,3$ années dans notre service. Le parcours professionnel des médecins correspondait à une pratique de six et sept ans de médecine interne et celui des infirmières respectivement à 7,10 et 11 ans de carrière professionnelle.

\section{Méthodes}

\section{La formation à la résilience}

La formation continue des soignants du service à l'éducation thérapeutique s'appuie sur une approche centrée sur le patient avec ses connaissances, compétences, croyances et comportements ${ }^{15}$. Cette formation de deux heures par semaine a été orientée vers une plus grande prise en compte des thèmes de la résilience et de l'empowerment. Sur un total de 40 séances de formation, 10 ont été dévolues spécifiquement aux théories de l'apprentissage, de l'empowerment et de la résilience. Les ateliers en éducation thérapeutique du patient ont permis aux soignants de s'entraîner aux entretiens centrés sur le patient, à l'empathie, à la reformulation, aux questions ouvertes et aux techniques d'entretiens motivationnels ${ }^{16}$. Les colloques ont aussi été organisés pour l'apprentissage du diagnostic pédagogique et de la compréhension du patient à l'aide d'une dizaine de grilles de lecture psychopédagogique et sociale ${ }^{17}$. Six colloques ont été organisés autour des concepts d'empowerment et de powerlessness. Finalement, quatre colloques ont été organisés autour du thème de la résilience en utilisant le modèle de la casita ${ }^{7}$ de Vanistendael. Le modèle psychosocial de la " casita " proposé par Vanistendael a été utilisé comme outil pour détecter et mettre en évidence les ressources des patients. Les res- sources du patient sont classées par le soignant en plusieurs catégories. Celles des liens familiaux et amicaux permet de rechercher notamment le tuteur de résilience. Le deuxième grand sous-groupe de ressources comprend le sens que l'on donne à la vie ainsi que les projets de vie. Finalement, parmi les ressources à mettre en évidence, le soignant peut rechercher les connaissances, compétences, qualités, hobbies et plaisirs du patient. La formation comprend également l'observation de tables rondes de patients animées par une psychologue. Ces tables rondes hebdomadaires ont aussi été plus orientées autour de la résilience. Ainsi, la psychologue invite les patients à s'exprimer sur le vécu de leur maladie, leurs difficultés et souffrances, mais surtout favorise une prise de conscience de leurs ressources et de leurs capacités à faire face à leur maladie.

Pour permettre au patient d'entrer dans un processus d'empowerment, les trois concepts suivants ont été abordés au cours de l'année. Tout d'abord, le concept d'une « prise de contrôle ", d'autonomie, de maitrise de soi est primordial pour entamer un processus de réconciliation identitaire. Ensuite, le concept du "lâcher prise ", du besoin de cohérence, permet au patient de commencer un processus de séparation identitaire. Finalement, le troisième concept aborde le besoin de relation " être en lien " qui induit un processus de sécurisation de soỉ.

\section{Protocole}

Trois entretiens d'entrée ont été menés par chacun des deux médecins. Les anamnèses d'entrée au début de la semaine sont toujours effectuées en binôme formé d'un médecin et d'une infirmière afin d'augmenter l'interdisciplinarité de l'entretien. Cette approche permet aussi d'éviter que les questions soient posées à deux reprises et les informations données par le patient sont immédiatement partagées par les soignants. Ainsi, six entretiens d'entrée ont été effectués par deux médecins et trois infirmières, ces dernières n'effectuant que deux entretiens. Les patients, diabétiques, sont ensuite suivis pendant une semaine pour une éducation thérapeutique complète. Ces entretiens ont un but d'anamnèse médicale typique, associé à un diagnostic pédagogique et à une anamnèse psychosociale. Six entretiens similaires, avec les mêmes soignants en binôme, ont été répétés 11 mois plus tard, après la formation complète. Bien entendu, les entretiens ont été effectués avec d'autres patients diabétiques venant aussi pour une semaine d'éducation thérapeutique. Ces entretiens ont été vidéo-enregistrés après consentement écrit du patient. Patients, infirmières et médecins ignorent aux deux temps, quels sont les critères ou contenus recherchés dans ces enregistrements. La responsable de la formation en psychopédagogie et éducation du patient n’a pas été impliquée dans l'analyse des cassettes vidéos. 


\section{Recherche et Perspectives}

\section{Analyse des entretiens \\ Elaboration de l'outil d'évaluation}

Les 12 cassettes (six enregistrées avant et six après la formation) ont été confiées, toutes en même temps, à une pédagogue, non impliquée dans ce projet de formation. Elle les a visionnées une première fois dans une visée exploratoire, à la recherche de critères lui permettant de construire la grille d'analyse des entretiens. Les grilles utilisées ont été validées et utilisées en éducation thérapeutique ${ }^{18}$ et la composante ressource de cette nouvelle grille a été inspirée de la grille « casita " validée par Vanistendael".

Les critères de construction de la grille sont les éléments repérés comme récurrents dans la façon qu'ont ces soignants d'animer les entretiens. Ces éléments ont été classés en deux catégories : d'une part, les questions posées par les soignants (Tableau 1) et, d'autre part, les attitudes adoptées en réaction à la réponse des patients (Tableau 2). La pédagogue a visionné une deuxième fois tous les entretiens (12) et les a analysés avec cette nouvelle grille comprenant des items bio-psycho-sociaux, avec un accent particulier sur la recherche des ressources du patient. L'analyse des entretiens en binôme a été faite spécifiquement et séparément pour chacun des soignant (médecin-infirmière).

Les questions recherchent : a) des informations d'ordre médical comme la date de début de la maladie, l'existence d'autres problèmes de santé, la survenue d'hypoglycémies, etc. ; b) des informations d'ordre thérapeutique comme le traitement, le régime poursuivi, etc. ; c) des informations d'ordre social comme la profession, la composition de la famille, etc. ; d) des informations sur la vie quotidienne comme l'organisation de la journée, la participation à telle activité, etc. ; e) des informations sur le vécu de la maladie et des traitements comme les injections, le régime, etc. ; f) des informations sur les connaissances et croyances concernant la maladie ou son traitement et $\mathrm{g}$ ) des informations sur les ressources personnelles ou environnementales $\mathrm{du}$ patient. Ces dernières sont particulièrement importantes pour repérer les ressources selon la classification de Vanistendael'. L'utilisation de la grille de la casita permet de rechercher les liens familiaux, sociaux, le sens de la vie et les projets, les compétences et hobbies et finalement les qualités de la personnalité du patient.

Les attitudes adoptées par les soignants en réaction aux réponses des patients ont été les suivantes : nouvelle question à la recherche d'informations biomédicales supplémentaires, apport au patient d'explications médicales ou thérapeutiques, félicitations et valorisation du patient, reformulation des propos du patient, incitation du patient à expliquer d'avantage son expérience, notification au patient de l'une de ses ressources.

\section{Administration de l'outil d'évaluation et analyse statistique}

Les statistiques ont été effectuées à l'aide du programme SPSS et les résultats sont exprimés en moyenne \pm SEM.

Les différences statistiques entre avant et après formation sont données par Sig-1 tailed et selon le test de Student.

\begin{tabular}{|l|c|c|c|c|c|c|}
\hline \multicolumn{7}{|c|}{ Tableau 1 : } \\
\multicolumn{1}{|c|}{$\begin{array}{c}\text { Nature des informations recherchées par les soignants avant et après la formation } \\
\text { en éducation thérapeutique du patient centrée sur la résilience et l'empowerment }\end{array}$} \\
\hline & \multicolumn{2}{|c|}{ Médecins } & \multicolumn{2}{c|}{ Infirmières } & \multicolumn{2}{c|}{ Population totale } \\
\cline { 2 - 8 } & Avant & Après & Avant & Après & Avant & Après \\
\hline Médicales & $13,1 \pm 3,0$ & $5,6 \pm 1,7 *$ & $2,6 \pm 1,1$ & $1,6 \pm 0,8$ & $7,9 \pm 2,2$ & $3,6 \pm 1,0^{*}$ \\
\hline Thérapeutiques & $3,5 \pm 0,8$ & $4 \pm 0,8$ & $1,6 \pm 0,5$ & $1,1 \pm 0,1$ & $2,5 \pm 0,5$ & $2,5 \pm 0,6$ \\
\hline Sociales & $1,8 \pm 0,4$ & $3,1 \pm 0,7$ & $0,8 \pm 0,3$ & $1,1 \pm 0,4$ & $1,3 \pm 0,3$ & $2,1 \pm 0,5$ \\
\hline Sur la vie quotidienne & $1,3 \pm 0,4$ & $2,3 \pm 1,0$ & $2,0 \pm 0,6$ & $1,1 \pm 0,3$ & $1,6 \pm 0,3$ & $1,7 \pm 0,5$ \\
\hline Sur les connaissances et croyances & $2,3 \pm 0,8$ & $3,5 \pm 0,6$ & $2,0 \pm 0,5$ & $1,0 \pm 0,4$ & $2,1 \pm 0,4$ & $2,2 \pm 0,5$ \\
\hline Sur le vécu de la maladie & $1,8 \pm 1,7$ & $0,8 \pm 0,3$ & $1,0 \pm 2,0$ & $0,3 \pm 0,2 *$ & $1,4 \pm 0,3$ & $0,5 \pm 0,1 *$ \\
\hline Sur les ressources & $0,1 \pm 0,1$ & $2,5 \pm 0,4^{* * *}$ & $0,1 \pm 0,1$ & $1,6 \pm 0,4^{* *}$ & $0,1 \pm 0,1$ & $2,0 \pm 0,3^{* * *}$ \\
\hline Moyenne \pm SEM ${ }^{*} \mathrm{p}<0,05 ;{ }^{* *} \mathrm{p}<0,01 ;{ }^{* * *} \mathrm{p}<0,001$ entre avant et après formation & \\
\hline
\end{tabular}




\begin{tabular}{|c|c|c|c|c|c|c|}
\hline \multicolumn{7}{|c|}{$\begin{array}{l}\text { Tableau } 2 \text { : } \\
\text { Comportements des soignants en fonction de la réponse des patients avant (T1), et après (T2), } \\
\text { la formation en éducation thérapeutique du patient centrée sur la résilience et l'empowerment }\end{array}$} \\
\hline & \multicolumn{2}{|c|}{ Médecins } & \multicolumn{2}{|c|}{ Infirmières } & \multicolumn{2}{|c|}{ Population totale } \\
\hline & $\mathrm{T} 1$ & T2 & $\mathrm{T} 1$ & T2 & $\mathrm{T} 1$ & T2 \\
\hline $\begin{array}{l}\text { Nouvelle question à la recherche } \\
\text { d'informations biomédicales }\end{array}$ & $21,6 \pm 3,9$ & $8,6 \pm 0,9 * *$ & $11,0 \pm 1,3$ & $2,6 \pm 0,6^{* * *}$ & $16,3 \pm 2,5$ & $5,6 \pm 1,0^{* * *}$ \\
\hline $\begin{array}{l}\text { Apport d'informations médicales } \\
\text { ou thérapeutiques }\end{array}$ & $1,8 \pm 0,4$ & $2,5 \pm 0,7$ & $3,3 \pm 0,9$ & $0,8 \pm 0,5^{*}$ & $2,5 \pm 0,5$ & $1,6 \pm 0,5$ \\
\hline Reformulation & $1,1 \pm 0,6$ & $4,5 \pm 1,1^{*}$ & $1,8 \pm 0,7$ & $4,1 \pm 1,7$ & $1,5 \pm 0,4$ & $4,3 \pm 0,9^{* *}$ \\
\hline Félicitations & $0,3 \pm 0,2$ & $0,3 \pm \pm 0,2$ & $0,6 \pm 0,4$ & $1,0 \pm 0,6$ & $0,5 \pm 0,2$ & $0,6 \pm 0,3$ \\
\hline $\begin{array}{l}\text { Incitation du patient à s'expliquer } \\
\text { les faits relatés }\end{array}$ & $2,3 \pm 0,9$ & $4,5 \pm 1,3$ & $3,1 \pm 1,1$ & $0,6 \pm 0,3^{*}$ & $2,7 \pm 0,7$ & $2,5 \pm 0,8$ \\
\hline $\begin{array}{l}\text { Commentaire mettant } \\
\text { en évidence les ressources } \\
\text { mentionnées par des patients }\end{array}$ & $0,1 \pm 0,1$ & $2,1 \pm 0,5^{* *}$ & $0,6 \pm 0,2$ & $1,0 \pm 0,2$ & $0,4 \pm 0,1$ & $1,5 \pm 0,3^{* *}$ \\
\hline
\end{tabular}

\section{Résultats}

\section{Avant formation}

Les soignants s'adressent essentiellement aux patients sous la forme de questions à la recherche d'informations médicales et/ou thérapeutiques. D'une part, ces questions initient l'échange avec le patient. D'autre part, elles relancent rapidement l'entretien après la réponse du patient. Les questions à la recherche d'informations médicales sont particulièrement nombreuses chez les médecins $(13,1 \pm 3,0)$ alors que la recherche d'informations sur les ressources est quasi inexistante $(0,1 \pm 0,1)$, chez les médecins comme chez les infirmières.

\section{Après formation}

Les professionnels posent moins de questions à la recherche d'informations médicales ou thérapeutiques $(6,2 \pm 1,5$ après, $10,5 \pm 2,5$ avant, $\mathrm{p}<0,05)$ et plus de questions sur la vie quotidienne en lien avec la maladie et le traitement, les connaissances et croyances et les ressources du patient $(15,5$ \pm 3,7 après, 6,7 $\pm 1,0$ avant, $\mathrm{p}<0,05$ ) (Tableau 3 - Figure 1). Les résultats les plus significatifs sont la diminution du nombre de questions biomédicales posées par les médecins $(5,6 \pm$ $1,7$ après, $13,1 \pm 3,0$ avant, $\mathrm{p}<0,05)$ et l'augmentation des questions sur les ressources des patients, chez les médecins $(2,5 \pm 0,4$ après, $0,1 \pm 0,1$ avant, $\mathrm{p}<0,001)$ et chez les infirmières $(1,6 \pm, 4$ après, $0,1 \pm 0,1$ avant, $\mathrm{p}<0,01)$.
Après la réponse du patient, de façon générale, les professionnels ne se recentrent plus aussi systématiquement sur la maladie en cherchant ou en donnant des informations médicales complémentaires (7,3 $\pm 1,4$ après, 18,9 $\pm 2,5$ avant, $\mathrm{p}<0,001)$. Ils adoptent davantage une attitude d' $\mathrm{em}$ powerment $(9,1 \pm 1,5$ après, 5,1 $\pm 0,9$ avant, $\mathrm{p}<0,05)$.

Le nombre de questions à la recherche d'informations biomédicales complémentaires diminue chez les médecins $(8,6$ $\pm 0,9$ après, $21,6 \pm 3,9$ avant, $p<0,01)$ et chez les infirmières $(2,6 \pm 0,6$ après, $11,0 \pm 1,3$ avant, $\mathrm{p}<0,001)$. Les infirmières réduisent leur apport d'informations biomédicales $(0,8 \pm 0,5$ après, 3,3 $\pm 0,9$ avant, $\mathrm{p}<0,05)$. Les reformulations des propos du patient sont beaucoup plus fréquentes (4,3 $\pm 0,9$ après, $1,5 \pm 0,4$ avant, $\mathrm{p}<0,01)$, de même que la signification au patient de ce qui, dans son discours, apparaît comme une ressource identifiée ou potentielle $(1,5 \pm 0,3$ après, $0,4 \pm 0,1$ avant, $\mathrm{p}<0,01)$. Ces dernières modifications sont plus marquées chez les médecins. La durée des entretiens n'est pas significativement modifiée avant et après (durée moyenne : 40,8 \pm 3 après, 38,3 minutes \pm 4 avant).

\section{Discussion}

Après cette formation en éducation thérapeutique au cours de laquelle un accent particulier a été mis sur les concepts et principes de résilience et d'empowerment, les soignants 


\section{Recherche et Perspectives}

Tableau 3 :

Questions des soignants et réactions aux paroles des patients avant et après la formation en éducation thérapeutique du patient centrée sur la résilience et l'empowerment

\begin{tabular}{|c|c|c|}
\hline & Avant & Après \\
\hline Questions à la recherche d'informations médicales ou thérapeutiques & $10,5 \pm 2,5$ & $6,2 \pm 1,5^{*}$ \\
\hline $\begin{array}{l}\text { Questions à la recherche d'informations sociales, sur la vie quotidienne, } \\
\text { les connaissances/croyances, le vécu de la maladie, les ressources }\end{array}$ & $6,7 \pm 1,0$ & $15,5 \pm 3,7$ \\
\hline Après réponse du patient recherche ou apport d'informations supplémentaires & $18,9 \pm 2,5$ & $7,3 \pm 1,4^{* * *}$ \\
\hline $\begin{array}{l}\text { Après réponse du patient, attitudes d'empowerment } \text { : reformulation, félicitation, } \\
\text { incitation du patient à s'expliquer les faits, commentaire sur les ressources }\end{array}$ & $5,1 \pm 0,9$ & $9,1 \pm 1,5^{*}$ \\
\hline
\end{tabular}

Figure 1 :

Questions des soignants et réactions aux paroles des patients avant et après la formation

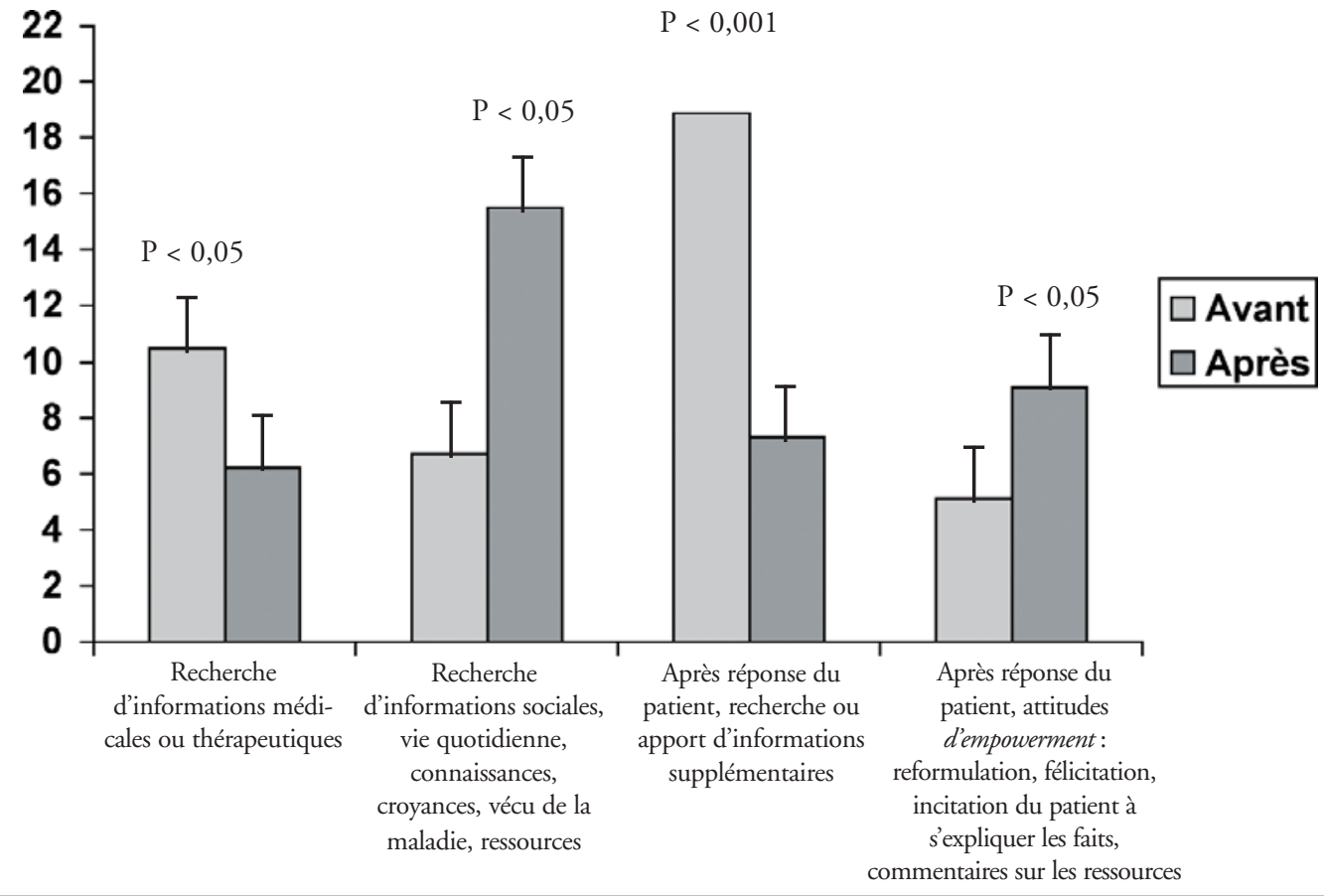

interrogent les patients sur leurs ressources personnelles ou environnementales alors que ce thème est pratiquement inexistant dans les entretiens menés avant cette formation. Il faut tout de même relever que ces entretiens médicaux ont été effectués dans un service d'éducation thérapeutique spécialisé et que, malgré cela, les ressources du patient étaient insuffisamment recherchées. De la même façon, ils

deviennent attentifs à la survenue, dans le discours du patient, d'éléments révélant une ressource potentielle et la mettent en lumière. Les soignants investissent donc effectivement plus souvent cette fonction de révélation et de valorisation des ressources existantes du patient pour lui permettre de les identifier et de les utiliser dans une meilleure gestion de sa vie avec cette maladie. Lidée sous-jacente est 
que prendre conscience de ses ressources et capacités à faire face est propre à augmenter son sentiment de maîtrise et de confiance en soi, et par là à encourager l'individu malade à prendre une part plus active dans sa prise en charge thérapeutique. Ainsi le patient diabétique, en rupture d'un sentiment d'identité et de sécurité (powerlessness), va pouvoir s'engager dans les trois processus d'empowerment. A l'aide de l'entretien centré aussi sur les ressources du patient, le soignant va lui permettre d'avoir un meilleur besoin de mầtrise (prise de contrôle), avec un meilleur besoin de cohérence (lâcher prise) associé à un besoin de relation (être en lien) ${ }^{3}$. Cette attention plus grande portée aux ressources du patient était effectivement le résultat souhaité. Il est cependant satisfaisant de constater que les soignants s'approprient suffisamment ces concepts pour que cela se traduise dans leur activité clinique quotidienne, en dehors d'une activité pédagogique formalisée. Les modifications de comportement des soignants sont plus larges. On constate que les questions posées portent plus sur les aspects sociaux et psychocognitifs et moins sur les aspects médicaux, centrant ainsi davantage l'anamnèse sur la personne porteuse de la maladie que sur la maladie elle-même. De façon générale, les soignants modifient assez profondément leur façon de mener ces entretiens. En effet, avant la formation, ils font suivre la réponse du patient par une nouvelle question à la recherche d'éléments biomédicaux supplémentaires. Il s'agit effectivement de la façon la plus habituelle de mener un entretien d'anamnèse où l'on cherche à confirmer et/ou infirmer la présence d'éléments listés pour orienter la démarche diagnostique. Cette finalité oblige à des modalités d'entretien de type «interrogatoire " avec une succession de questions/réponses, qui renforce les patients dans une attitude passive. Malheureusement, les formations des soignants sont trop focalisées sur les aspects biomédicaux. Peu de place est donnée à l'individu-patient et à ses besoins, et surtout à ses ressources pour construire avec lui son programme de soins. Après la formation, la réponse du patient est moins systématiquement suivie d'une nouvelle question mais plus souvent d'une reformulation. Cela incite le patient à expliciter, clarifier ou argumenter d'avantage ses propos et l'aide à se positionner dès le premier entretien comme partie prenante dans l'élaboration de cet état des lieux, base de son projet thérapeutique. Cette attitude plus active des patients est nécessaire pour qu'ils puissent gérer leur maladie chronique. En effet, c'est en dehors de toute présence médicale que les patients diabétiques doivent assumer la plupart des soins et adapter leurs comportements de santé.

On remarque que le temps d'entrevue reste inchangé alors même que des domaines nouveaux, comme celui des ressources, sont investigués et qu’une place plus grande est faite à l'expérience du patient. Cela mérite d'être mis en exergue car une objection fréquente faite à l'adoption d'une approche centrée sur le patient est celle de rallonger le temps de la consultation. Ces résultats confirment les travaux de Lacroix et Assa ${ }^{19}$ qui montrent que lorsque les soignants sont formés à l'éducation thérapeutique, le temps de parole du patient augmente alors que celui du soignant diminue. Cela n'est possible que parce que les soignants posent moins de questions à la recherche d'informations d'ordre médical ou thérapeutique. Les informations biomédicales recherchées sont plus ciblées, un grand nombre d'entre elles pouvant être retrouvées dans les dossiers et les courriers médicaux. Les résultats de cette étude montrent un changement important dans la pratique des soignants. Elle est certainement due à de nombreux facteurs associés. La formation hebdomadaire comprenait des ateliers centrés aussi bien en éducation thérapeutique que sur l'empowerment. Il semble difficile de distinguer et d'évaluer séparément l'impact de ces différentes parties de la formation. La durée de la formation, la fréquence des séances, et son ancrage dans une pratique quotidienne au sein d'une équipe dont c'est la culture, facilitent sans doute ces transformations ${ }^{20,21}$. Les modifications de comportements chez les soignants ne témoignent pas seulement de l'adoption de nouvelles modalités d'entretien, mais aussi de l'évolution vers un nouveau paradigme : alors qu'habituellement il est attendu de la personne malade qu'elle applique au plus près les directives des professionnels de santé, il est proposé dans cette approche qu'elle participe activement à la construction et à l'évolution de son projet thérapeutique, à l'aide de ses propres ressources. Or Anderson ${ }^{22}$ témoigne de la difficulté des soignants à adopter des paradigmes différents de ceux auxquels ils ont souscrit au cours de leur formation initiale. Changer son rôle d' " expert " pour endosser le rôle de « facilitateur " est un passage difficile ${ }^{23}$. L'objectif suivant, en cours de réalisation, est évidemment d'évaluer le retentissement de ces changements sur l'attitude des patients.

\section{Conclusions}

L'approche diagnostique traditionnelle n’a pas été conçue pour aider les patients à repérer leurs ressources personnelles ou environnementales, sur lesquelles ils pourraient s'appuyer pour faire face à la maladie chronique et poursuivre leur vie avec une qualité optimale. Une formation des soignants en éducation thérapeutique des patients, axée sur les concepts de résilience et d'empowerment, a permis à des médecins et des infirmières d'être plus à l'écoute et à la recherche des ressources des patients dans leurs entretiens d'anamnèse. Mais au-delà, cette sensibilisation a ouvert les soignants à une approche différente, davantage centrée sur 


\section{Recherche et Perspectives}

le patient-individu. Porter leur regard sur ce qui va bien, et pas uniquement sur ce qui est malade, a amené les soignants à modifier profondément leur approche, les faisant passer d'une logique d'interrogatoire à une logique d'incitation des patients à prendre une part active à la compréhension et à la construction de leur propre prise en charge dès la première rencontre.

\section{Références}

1. Golay A, Lagger G, Giordan A. Une éducation thérapeutique en quatre dimensions pour diminuer la résistance au changement des patients obèses. Médecine des Maladies Métaboliques. 2007;1:69-76.

2. Golay A, Lagger G, Chambouleyron M, Carrard I, LasserreMoutet A. Therapeutic patient education in diabetes. Diabetes Metab Res Rev 2007, in press.

3. Aujoulat I, d'Hoore W, Deccache A. Patient empowerment in theory and practice: Polysemy or cacophony? Pat Educ Counsel 2007;66:13-20.

4. Manciaux M, Tomkiewicz S, La résilience aujourd'hui. In Gabel M, Jesu F, Manciaux M. Bientraitances. Mieux traiter familles et professionnels. Paris :Editions Fleurus, 2000:31340.

5. Werner E. The children of Kauai: resiliency and recovery in adolescence and adulthood. J Adolesc Health 1992;13:262-8.

6. Tomkiewicz S, Eunson PD, Kreisler L. L'enfant et la guerre, Enfance majuscule 1996;31:3-34.

7. Vanistendael S, Lecomte J. Le bonheur est toujours possible. Construire la résilience. Paris: Bayard, 2000.

8. Forrest CB. Outcomes research on children, adolescents, and their families: directions for future inquiry. Med Care 2004;42(4 Suppl) III19-23.

9. Young-Saleme TK, Prevatt FF. Brief report: psychometric evaluation of the severity of illness scale in a pediatric oncology sample. J Pediatr Psychol 2001;26:55-60.

10. Mangelli L, Gribbin N, Buchi S, Allard S, Sensky T. Psychological well-being in rheumatoid arthritis : relationship to 'disease' variables and affective disturbance. Psychother Psychosom 2002;71:112-6.

11. Vanistendael S. "Resilience and spirituality" in Monroe B, Oliviere D, Resilience in palliative care - achievement in adversity. New York: Oxford University Press, 2007:11536.

12. Michaud PA. La résilience: un regard neuf sur les soins et la prévention. Arch Pédiatr 1999;6:827-31.

\section{Contributions}

Monique Chambouleyron a rédigé la version initiale $\mathrm{du}$ manuscrit. Aline Lasserre-Moutet a effectué le recueil des données et interprété les résultats. Stefan Vanistendael a participé à la conception du protocole de recherche. Grégoire Lagger a contribué à la discussion des résultats. Alain Golay a participé à la conception du protocole de recherche et à la révision du manuscrit.

13. Dayer-Metroz MD. Resilience: a concept covering new perspectives on long-term patient care. Rev Med Suisse Romande 2002;122:245-7.

14. Golay A, Lagger G, Giordan A. Motivating patients with chronic diseases. Journal of Medicine and the Person 2007:5:57-63.

15. Assal JP, Golay A. Patient education in Switzerland: from diabetes to chronic disease. Patient Educ Couns 2001;44:65-9.

16. Miller WR, Rollnick S. Motivational interviewing. Preparing people to change addictive behaviors. The Guilford Press - New York, 1991(2nd edition), 2002.

17. Sommer J, Gache P, Golay A. L'enseignement thérapeutique et la motivation du patient. In: Richard C, Lussier MT (dir.) La communication professionnelle en santé. Québec :ERPI, 2005:656-91.

18. Jaquemet S, Lacroix A, Perolini M, Golay A, Assal JP. Qualitative evaluation of courses intended for patients suffering from chronic diseases. New observation method for the continuous training of the healthcare team. Pat Educ Counsel 1998;34:201-12.

19. Lacroix A, Assal JP. Therapeutic Education of Patients New approaches to chronic illness. ParisEditions Maloine, 2003.

20.Woodcock AJ, Kinmonth AL, Campbell M, Griffin SJ, Spiegal NM. Diabetes care from diagnosis: Effects of training in patient-centerd care on beliefs, attitudes and behaviour of primary care professionals. Patient Educ Couns 1999;37:6579.

21. Doherty Y, Hall D, James PT, Roberts SH, Simpson J. Change counselling in diabetes: the development of a training programme for the diabetes team. Patient Educ Couns 2000;40:263-78.

22. Anderson RM, Funnell MM. Patient impowerment :reflections on the challenge of fostering, the adoption of a new paradigm. Patient Educ Couns 2005;57:153-7.

23. Adolfsson ET, Smide B, Gregeby E, Fernström L, Wikblad $K$. Implementing empowerment group education in diabetes. Patient Educ Couns 2004;53: 319-24.

Manuscrit reçu le 7 décembre 2006 ; commentaires éditoriaux formulés le 14 septembre 2007 ; accepté pour publication le 12 octobre 2007. 